\title{
Vaccine Potentiality and Stem Cells Enhancing Cure to Cancer
}

Parthasarathi D*and Palki D

Department of Biotechnology \& Bioscience, Fakir Mohan University, Balasore, Odisha, India

\begin{abstract}
Vaccine potentiality and stem cell therapy is the new development in curing the Cancer. There are different types of cancer and different curing methods are being discussed by different scientist. The new diagnostic methods using DNA vaccine, adjuvants and target oriented cells methods are used in a descriptive method. The stem cell therapy also enhancing in identifying the site of tumour regenesis and curing the tumour by inducing different target oriented cells like chemokines and interleukin (IL). Beside these mesenchymal stem cells also regenerates the new cell lines for new target oriented cells. Hence no doubt at molecular level, the alteration of stem cell self-renewal pathways has been recognized as an essential step for cancer stem cells transformation. Influencive study and therapy will help to eradicate the disease and vaccines or stem cells will be new era in pharmaceuticals for Cancer.
\end{abstract}

Keywords: Tumours; Cancer; Target cells; Vaccine; Stem cells

\section{Introduction}

"Cancer", known medically as a malignant neoplasm [1] which causes due to abnormal growth of normal cells. The development of cancer starts from damages made to the normal cells DNA [2]. Whenever the DNA is damaged the body tries to recover the damage part and form new DNA repair part. On forming the new cells the damaged portion of the cells are also utilized by the new normal cells. This starts the abnormal growth of cells which forms "Tumours".

\section{Tumours and myeloma cells}

Tumours are abnormal growth of cells which may be cancerous or may not be cancerous [3]. Cancerous cells are malignant tumours and non cancerous cells are benignant tumours. Nucleophosmin belongs to the family of nuclear chaperones, nucleoplasmins [4]. Tumour protein 53 is a tumor suppressor protein in humans, encoded by the TP53 gene. p53 is crucial in multi cellular organisms, where it regulates the cell cycle and functions as a tumor suppressor that is involved in preventing cancer.The TP53 gene, which encodes $p 53$, is one of the most frequently mutated genes in human cancers [5]. Tumour stroma consists of various inflammatory cells like lymphocytes, macrophages, neutrophils, plasma cells, mast cells and eosinophils. The inflammatory cells in tumour stroma are result of host response to tumour cells. Recently, attention has been directed towards tumour associated tissue eosinophils and mast cells and their role in the biologic behavior of tumours [6]. Tumour cells in which not only overexpression of nucleophosmin leading to a decrease in cell sensitivity but also to apoptosis [4]. If the cancer is in one location, the goal of treatment is usually to remove the tumor with surgery. If the tumour has spread to local lymph nodes only, sometimes these can also be removed. If all of the cancer cannot be removed with surgery, the options for treatment include radiation and chemotherapy, or both. Some patients need a combination of surgery, radiation and chemotherapy.

Myeloma cells also known as plasma cell myeloma or Kahler's disease are a cancer of plasma cells, a type of white blood cells normally responsible for the production of antibodies. The collection of abnormal cells near the bone causes bone lesions which interferes normal blood cells. Interferes production of the paraprotein, an abnormal antibody which causes kidney problems. Hypercalcemia also results in this type of cells [7].

\section{Stem cells}

Stem cells are defined as cells that have the ability to perpetuate themselves through self renewal and to generate mature cells of a particular tissue through differentiation. Stem cell biology provides new insights into cancer biology [8]. The relationship between the stem cells and cancer cells through light on many aspects like self renewal, potentiality and mechanism. Monocytes and their progeny, as myeloidderived cells, promote lymphomagenesis by suppression of host immunity, stimulation of tumor angiogenesis and provision of tropic factors. Thus an introduction of biomarkers is used [9].

Tumor stem cells were first isolated as clones based on the soft agar cloning technique. However, this technique is time and labor intensive and only a small fraction of primary tumours yield adequate numbers of colonies. Undifferentiated tumor stem cells sphere culture have been derived from primary tumours \& cell lines using serum- free medium that is supplemented with growth factors, such as epidermal growth factor \& basic fibroblast growth factors. Serum replacement results in the morphological change of adherent monolayer culture to tumor stem cells - enriching floating spheres, such as neurospheres [8].

\section{Target cells}

Target cells are cells which have the antigenic peptide combined with MHC class I (Major Histocompatibility Complex) molecules to Cytotoxic T cells. These cells have MHC molecules on their surface (in contrast to Antigen, which in addition also have MHC class II molecules). Target Cells are the normal cells of the body which have undergone some alteration and synthesize abnormal proteins e.g. tumor cells, virus infected cells [10].

*Corresponding author: Parthasarathi D, Department of Biotechnology \& Bioscience, Fakir Mohan University, Balasore, Odisha, India, E-mail: parthasarathi5ds@yahoo.co.in

Received December 02, 2011; Accepted December 20, 2011; Published December 22, 2011

Citation: Parthasarathi D, Palki D (2011) Vaccine Potentiality and Stem Cells Enhancing Cure to Cancer. J Cancer Sci Ther S17. doi:10.4172/1948-5956. S17-008

Copyright: (C) 2011 Parthasarathi D, et al. This is an open-access article distributed under the terms of the Creative Commons Attribution License, which permits unrestricted use, distribution, and reproduction in any medium, provided the original author and source are credited. 


\section{Vaccination}

A vaccine is a biological preparation that improves immunity to a particular disease. A vaccine typically contains an agent that resembles a disease-causing microorganism, and is often made from weakened or killed forms of the microbe, its toxins or one of its surface proteins. The agent stimulates the body's immune system to recognize the agent as foreign, destroy it, and "remember" it, so that the immune system can more easily recognize and destroy any of these microorganisms that it later encounters [11].

Cancer vaccine refers to a vaccine that prevents infections with cancer-causing viruses, treats existing cancer or prevents the development of cancer in certain high risk individuals. Some types of cancer, such as cervical cancer and some liver cancers are caused by viruses, and traditional vaccines against those viruses, such as HPV vaccine and Hepatitis B vaccine, will prevent those cancers [12]. Human papillomaviruses (HPVs) are small DNA tumor viruses, some of which induce malignancy in genital, anal, head and neck and also skin tissues [13]. Different immunoglobins acts as monoclonal antibody [14], Gardasil vaccine, Cervarix vaccine for HPV [15].

\section{Tumour Cell Growth}

Normal cells when get hampers by some virus,bacteria or any manipulation of the genetic material i.e. DNA, the body immediately regenerates its self repairing activity on generating new cells. As the cell repairs new cells generated. The repair system duely absorb the degenerated cells along with releasing new abnormal cells which gives rise to tumours which may be cancerous or may not be harmful. Cancer occurs after a single cell in a tissue is progressively genetically damaged to produce a cancer stem cell possessing a malignant phenotype. These cancer stem cells are able to undergo uncontrolled abnormal mitosis, which serves to increase the total number of cancer cells at that location. When the area of cancer cells at the originating site becomes clinically detectable, it is called a primary tumor. Some cancer cells also acquire the ability to penetrate and infiltrate surrounding normal tissues in the local area, forming a new tumor. The newly formed "daughter" tumor in the adjacent site within the tissue is called a local metastasis [16]. Cancer may cause in any body parts whether its skin, oral parts, internal parts or body fluid.

Melanin (skin pigment) is an amorphous, irregular polymer composed of intimate mixtures of two separated but biogenetically related pigments: eumelanins and pheomelanins. Melanoma is a poor prognosis skin cancer that affects about 150000 new patients per year in world. Although localized disease is frequently curable by surgical removal, there is no effective treatment for metastatic melanoma. Patients with metastatic malignant melanoma have an average median survival rate of 6 months and a 5-year survival rate of less than 15\% [17]. The cause of tumours is may be due to genes or hereditary or over exposure to sun etc lead to cancer.

Breast cancer incidence and mortality rates decrease with environmental conditions that promote Vitamin D synthesis in human skin including lower latitude and higher personal exposure to sunlight. Breast cancer incidence with changes in cloudiness, which decrease human Vitamin D synthesis [18]. During winter 1-2 months the synthesis of vitamin $\mathrm{D}$ is low compare to other seasons, as it recites deficiency of serum vitamin $\mathrm{D}$ due to low absorption of $\mathrm{UV}$ rays or exposure to sun rays. At the end of the season it is observed that the serum level is low from the compared results of summer or other seasons. Gain and loss of function assays have demonstrated a critical role for osteopontin in tumor metastatic function in colon, liver, and breast cancers [19]

Oral submucous fibrosis (OSF) is a high risk precancerous condition which was first described in the early 1950s characterized by changes in the connective tissue fibers of the lamina propria and deeper parts leading to stiffness of the mucosa and restricted mouth opening. Epidemiological and in vitro experimental studies have shown that chewing areca nut (Areca catechu) is the major aetiological factor for OSF. It is logical to hypothesize that the increased collagen synthesis or reduced collagen degradation as possible mechanisms in the development of the disease [20]. Beside this tobacco use and alcohol abuse are the dominant risk factors, human papilloma viruses (HPVs) have been found in a small proportion suggested an association between periodontal disease and the risk of various human malignant neoplasms [21].

Laryngeal tumours are among the most cancers in head and neck cancers. The incidence of these tumours is increasing due to the worldwide use of tobacco. Malignant tumours of the larynx arise from the surface epithelium and therefore are squamous cell carcinoma (SCC) or one of its variants is used as the therapy for cancer in head or neck [22].

Endocan also called endothelial cell-specific-molecule-1 is a product of endothelial cells, highly regulated by vascular endothelial growth factor and expressed during the switch between dormant to fast-growing angiogenic tumours. Endothelial cells in tumours may not have the appearance of normal endothelial cells as they are disorganized, loosely connected, branched, sprouting and forming a defective cellular lining of the vessel wall. [23].

The pro-inflammatory regulators such as chemokines and their receptors network seem to play crucial functions in prostate tumorigenesis, although chemokines have been thought of primarily as leukocyte attractants. In general, the function of chemokines is to arrest leukocytes at inflamed blood vessels and to lead them to specific sites of inflammation. Due to specific function of site-specific homing for leukocytes from inflammatory sites, these mediators may play several key steps of prostate carcinogenesis including production of inflammatory cells. Chronic inflammation predisposes cells to produce deregulated amounts of chemokines for malignant transformation and progression [24].

\section{Tumour Cell Toxicity}

The cancer cell toxicity increases when its normality reaches the abnormal stages by producing enzymes which are toxins to normal cells and causes death. Osteopontin (OPN) is a secreted phosphoprotein which plays a critical role in metastasis of colon, liver, and breast cancers. A substantial body of evidence indicates that OPN is a major regulatory factor in increased cellular migratory and invasive behavior, increased metastasis, protection from apoptosis, promotion of colony formation and 3D growth ability, induction of tumor-associated inflammatory cells, and induction of expression of angiogenic factors. The canonical pathway for OPN signaling focuses on its binding interactions with integrin and CD44 cell surface receptors [19]. Increased activation of the proto-oncogene c-Src (Src) has been established in enhancing tumor progression in human cancer and corresponds with poor clinical outcome. Src is responsible for governing signaling path- 
ways that regulate proliferation, angiogenesis, and resistance to apoptosis, adhesion, motility and invasion [25].

Many viruses have been associated with human breast cancers, including Epstein-Barr and Cytomegalovirus. New evidence has revealed the frequent presence of highrisk human papillomavirus (HPV) strains HPV16 and HPV18 in breast carcinoma biopsies. Oral biopsy reveals a comparatively low presence of HPV in pre-malignant oral lesions. HPV in a subset of breast cancers raises the question of whether, in addition to a role in carcinogenesis, HPV may also preferentially infect already developing tumours and subsequently mediate their growth. Beside this toxicity of hormonal balance, unlimited smoking and alcohols bring the chemical disturbances inside the body which brings the chance to HPV virus to infect and create carcinogenesis. [26]

Epidemiological and in vitro experimental studies have shown that chewing areca nut (Areca catechu) is the major aetiological factor for Oral submucous fibrosis (OSF). The major areca nut alkaloids are arecoline, arecadine, arecolidine, guyacoline and guacine. The important flavonoid components in areca nut are tannins and catechins. These alkaloids undergo nitrosation and give rise to $\mathrm{N}$-nitrosamine which might have cytotoxic effect on cells. The cytotoxic effect leads the body to produce the different interleukins and increases the chances of TGF (Tumour Growth Factor) [20].

Butyric acid (BA), an extracellular metabolite from periodontopathic bacteria, induces apoptosis in T cells, B cells and inflamed gingival fibroblasts. The role of BA as a histone deacetylase inhibitor and an epithelial cell migration-inducing factor. Podoplanin, a transmembrane glycoprotein, in all layers of oral sulcular and junctional epithelia in patients with chronic periodontitis. Podoplanin was originally discovered on the surface of podocytes in rats with puromycin-induced nephrosis as a $38-\mathrm{kDa}$ mucoprotein linked to flattening of foot processes. Enhanced expression of podoplanin has also been demonstrated at the invasive front of a number of human cancers including human squamous cell carcinoma. Podoplanin contributes to tumor metastasis through its platelet aggregation-inducing activity and to tumor invasion by binding erzin, radixin and moesin proteins, leading to activation of RhoA and remodeling of the actin cytoskeleton of tumor cells through epithelial-mesenchymal transition and connective cells. A recent study demonstrated that BA acts as a histone deacetylase inhibitor, and that periodontal disease could be as a risk factor for HIV-1 reactivation in infected individuals [21].

Liver metastatis caused from other primary tumours with particular interest in its effect on metastases from neuroendocrine tumours since they tend to be vascular in nature [27]. Chemokines in the inflammatory responses to prostate cancer initiation to metastasis process. Chemoattractant chemokines, more popularly known as CXC chemokines, and their receptors [24].

\section{Relevant Curing Methodologies}

Cancer screening and diagnosis is done by Pap test. The test was first developed by George Papanicolaou as a method in understanding the menstrual cycle. Later early detection of cervix, breast, colon, rectum, endometrium, prostate, thyroid, oral cavity, skin, lymph nodes, testes, and ovaries cancers were identified and practiced in the clinic [2]. Surgery plays an important role in removal of cancer, in the present scenario but there is also a chance that cancer may return even after the removal of the tumours or cancerous cells. The development in science is facing the challenge yet to demolish this cancer, to some extent it has delivered many new technologies and method but its success key is yet not properly been identified. There are many therapies and surgery routes to demolish cancer in the body. The side effects or after effects is too dangerous to survive for the patient.

Tumstatin (a3(IV)NC1) a $28 \mathrm{kDa}$ cryptic domain is liberated from the carboxy terminal region of a 3 chain type IV collagen by matrix metalloproteases was shown to inhibit proliferation of melanoma and epithelial tumor cell lines in-vitro by binding to CD47/aVb3/a3b1 integrin complex. Tumstatin binds to aVb3/a3b1 integrins and inhibits PI3K/ Akt/mTOR/ 4E-BP1/COX-2 signaling leading to the inhibition of protein synthesis and hypoxic tumor angiogenesis [28].

The biomedical devices being introduced by the researchers for the curing of cancer such as Bead Array Platform. It is basically software designed for microarray data to be pre-processed through various model-based statistical methods. Significant improvement accepts the summarized bead-type data. The software developed will facilitate molecular biomedical - especially cancer - research [29]. Similarly, the heat effect, biological effects of FIR (Far Infrared Ray) also cultured on hepatocellular carcinoma cells HepG2 to detect another cancer responsible for its anticancer effects [30]. Some patients suffer with prostate cancer they are treated with radiothereapy. The effectiveness of radiotherapy mainly depends on the molecular context of the tumor. $p 53$ tumor suppressor protein plays an important role in radiation response. Radiotherapy failure is more common in prostate cancer patients with abnormal $p 53$. Failure of radiotherapy is observed in about $30-40 \%$ of prostate cancer patients. The results of radiotherapy in prostate cancer could be improved. There are agents that act on the molecular level that may make this possible [31].

Some biological responses like Interferons (IFN) which modifies and may inhibit cancer cell growth by regulation of the immune response, inhibition of angiogenesis and direct antitumour activity. Interferon-gamma (IFN- $\gamma$ ), a member of the IFN family, has been associated with activation, growth, and differentiation of a variety of cells including T cells, B cells, macrophages, NK cells, endothelial cells, and fibroblasts [32]. Interactions initiate the classical HJanus kinaseH-STAT (HJAK-STATH) signalling pathway, whereas the early studies were performed with mixtures of IFNs extracted from white blood cells, most clinical trials have been performed with single-species recombinant IFN-a, and more recently with variants conjugated to poly-ethylene-glycol (PEGylation). PEGylation delays the elimination of IFN, allowing for once weekly treatment in humans and carries a promise of higher efficacy and reduced toxicity Although IFN is in some clinical use in advanced osteosarcoma, the relative contributions of direct antitumour effects, inhibition of angiogenesis and indirect immune-mediated effects are not known [33]. Metastasis a cure induced for the breast cancer regulates PTEN (phosphatase tensin) protein. This led us to believe that both the proteins might interact with each other but the gene involves during metastasis MTA1 (metastasis gene) involves itself to regulate the spread of cancer [34]. Drugs applied in vitro and in vivo results 50-60\% decrease in tumour cell growth. Drug like Hydroxyurea, Aphidicolin and Etoposide are some which inhibit the cancer formation tumours. Aphidicolin represents a good example of a potential anticancer drug that reached the stage of clinical trials. Hydroxyurea alone has been proven at early stage to be of no use for glioma treatment. Etoposide is a drug should have been successful for glioma treatment, but clinical trials proved to be of very limited use 
[35]. Nanotechnology also plays an important role in modern world for the treatment of cancer. It is basically the drug targeted process where the drug is induced particular to the target site of tumours. Liposomes and micelles seems promising with drug transfer to tumor cells within the tumor site. Immunomicelles containing a photosensitizing agent and tumor-specific monoclonal antibody have been successfully used in photodynamic therapy against murine lewis lung carcinoma [36].

\section{Antibody \& vaccine potentiality}

The application of immunological therapies against cancer is a null effect on tumor growth. The immunostimulatory hypothesis of cancer suggests that, upon certain circumstances, the growth of incipient and established tumours can be accelerated rather than inhibited by the immune response supposedly mounted to limit tumor growth [37]. Anti-Gal is the most abundant antibody in humans, constituting $1 \%$ of immunoglobulins. Anti-Gal binds specifically a-gal epitopes (Gala1$3 \mathrm{Gal}$ (1-4GlcNAc-R). Immunogenicity of autologous tumor associated antigens (TAA) is greatly increased by manipulating tumor cells to express $\alpha$-gal epitopes and bind anti-Gal. Glycolipids with agal epitopes ( $a$-gal glycolipids) injected into tumours insert into the tumor cell membrane. Anti-Gal binding to the multiple $\alpha$-gal epitopes de novo presented on the tumor cells results in targeting of these cells to APC via the interaction between the Fc portion of the bound anti-Gal and Fc $\gamma$; receptors on APC. The APC process and present immunogenic TAA peptides and thus, effectively activate tumor specific CD4+ helper $\mathrm{T}$ cells and CD8+ cytotoxic $\mathrm{T}$ cells which destroy tumor cells in micrometastases [38].

HLA-A2.1 is a prevalent human MHCI molecule. Many well characterized HLA-A2.1 restricted epitopes have been tested for their therapeutic effects for viral infection or tumor formation in the HLA-A2.1 transgenic mouse. HLA-A2.1 restricted epitopes and generate a multivalent epitope DNA vaccine. Multivalent DNA vaccine provided complete protection and strong therapeutic effect against infection with a single booster immunization. Five epitopes were immunogenic when tested in HHD mice by epitope and DNA vaccination. All five epitope DNA vaccines provided strong and specific protective immunity in HLA-A2.1 transgenic rabbits. HLA-A2.1 transgenic rabbits are more advantageous for screening and testing new protective and therapeutic DNA vaccines in vivo in a natural papillomavirus/host model [13].

The secretion of angiogenic stimulators by malignant cells activates endothelial cells to proliferate and form new blood vessels, and this additional vascular capacity is essential for tumor growth and metastasis. Various approaches used to target angiogenic vasculature, including monoclonal antibodies and synthetic molecules, which have been shown to inhibit tumor growth in animal models and have been administered in clinical trials. This vaccination approach offers the advantage that targeting of autologous antigens and previously uncharacterized antigens can be achieved without needing to isolate the relevant targets. The successful purification of the essential antigens will help for the administration of anti-cancer vaccines [39].

Adjuvant treatment of the cancer randomized adjuvant interferon alpha (IFN) or highdose interleukin 2 (IL-2), observation showed that within no delay in time the improvement in survival tissue is more. Observation remains standard care after nephrectomy, and eligible patients should be enrolled in clinical trials. Granulocyte-macrophage colony-stimulating factor (GM-CSF), IFN and IL-2 can stimulate large number of T-lymphocytes, antigen-presenting cells and can induct anti-tumor immunity in RCC (Renal cell carcinoma) patients [40].

Interferon (IFN $\gamma$ ) is a potent inhibitor of growth of mammalian cells. The IFN effects growth arrest through cellular functions of gene products, whose synthesis is induced or repressed by the IFN. IFN $\gamma$ found to induce or sensitise target cells to apoptosis through the induction of caspase 8 by IRF1 in Ewing tumor, neuroblastoma or medulloblastoma or by downregulating the levels of p21 in human hepatocellular carcinoma cells. The interferon transferred into the body with bovine serum or antibodies, antibiotics [41].

Target-specific anti-cancer drugs can be developed, if we can identify unique biomarkers that can differentiate these cancer cell-expressed immunoglobulins from those originated from normal B cells. RP215 is one of the three thousand monoclonal antibodies (Mabs) that has been generated against an ovarian cancer cell line, OC-3-VGH. This antibody has been found to react specifically with a carbohydrate-associated epitope in the variable regions of cancer cell-expressed immunoglobulins, designated in general as CA215 [14]. Similarly, tumour derived cytokines interact with their cognate receptors to activate the $\mathrm{NF}-\kappa \mathrm{B}$ transcription factor, which induces atrophy by activating the ubiquitin proteasome system to degrade the carcinoma cells [42]. Plant lectins are of great use in the detection of the complex carbohydrates of the cell membrane. Lectins are valuable cyto and histological tools for the localization of defined carbohydrate determinants. Horseradish peroxidase conjugated Canavalia gladiate lectin was used to evaluate its binding property to different types of leukemic cells and to investigate whether this lectin could be used for the typing of leukemias. This can be used with the monoclonal antibody for the curing process [43]. Cystatins are natural cysteine protease inhibitors with specific activities. The primary function of cystatins is to limit the cysteine protease activity released from lysosomes and produced during inflammation they may also interfere in tumor development and metastasis. Thus, an imbalance in the levels and activity of cathepsins B and L and the levels of cystatins, which are reduced in tumor cells, has been observed. The inhibitory effect of phytocystatins on human breast cancer invasion has been recently evaluated. Sugarcane cystatin, CaneCPI-4, showed strong inhibitory activity against cathepsins B and L and significantly reduced tumor cell invasion [44].

Bacterial protein toxins are among the most potent cell-killing molecules known. They bind to cell surface antigens, enter cells by endocytosis, reach the cytoplasm, and disrupt protein synthesis via interference with mRNA translation, leading to cell death usually via apoptosis. The first PE-based immunotoxins were composed of fulllength PE protein attached to whole monoclonal antibodies (MAbs). Recombinant DNA techniques enabled the development of singlechain recombinant immunotoxins. DNA sequences encoding only the antigen-binding site of the antibody were fused to DNA sequences encoding PE38, the truncated form of PE [45]. DNA vaccines have many advantages such as high stability, not being infectious, focusing the immune response to only those antigens desired for immunization and long-term persistence of the vaccine protection [46]. Monoclonal antibodies (e.g. trastuzumab, pertuzumab) and small molecule tyrosine kinase inhibitors (e.g. lapatinib, afatinib) have shown significant clinical responses in some breast cancer subtypes [47]. Monoclonal antibody to human $\mathrm{CD} 3$, when used in combination with other antibodies, is regarded as a reliable pan T-cell antibody used in the immunophenotyping of lymphomas in paraffin sections. Polyclonal antibody to CD3 immunoreactivity has also been reported in a minority of Reed- Stern- 
berg cells of Hodgkin's disease and in some B cell. The identification of such cases indicates that the use of CD3 antibody alone in paraffin sections may lead to an incorrect determination of cell lineage in some B-cell NHL. However, polyclonal CD3 is useful to distinguish between T-cell and B cell lymphomas when used in conjunction with CD20 [48]. Microbial adjuvant in vaccines activates key transcription factors, including NF- $\kappa \mathrm{B}$ and interferon response factors [49]. NSCLC vaccine (non-small-cell lung cancer ) III trials are investigating this approach in different treatment settings: the Melanoma AntiGEn A3 vaccine in resected early-stage NSCLC, the L-BLP25 vaccine in locally advanced NSCLC after chemoradiotherapy, and belagenpumatucel-L, the epidermal growth factor and the TG4010vaccine in advanced stage, either as an adjunct to chemotherapy or as maintenance after completion of chemotherapy [50][Figure 1]. Some of the renowned vaccines used in the cancer treatment such as:

- Gardasil and Cervarix preventive HPV vaccine [15]

- Microbial adjuvant

- NSCLC vaccine (non-small-cell lung cancer )

1. AntiGEn A3 vaccine

2. L-BLP25 vaccine

3. Belagenpumatucel-L vaccine

4. TG4010 vaccine

- Monoclonal antibodies

- Trastuzumab

- Pertuzumab

- DNA vaccines

\section{Stem cell therapies}

Asymmetric division, a process by which stem cells divides to gen- erate the diversity of cell types that populate adult organisms. Asymmetric division has been found in virtually all developing systems where stem cells need to simultaneously proliferate and generate differentiated cells: brain, skin, gut, mammary gland, and hematopoiesis of mammals [51]. Cancer Stem cells (CSCs) are the source of many solid tumor types including hepatocellular carcinoma. MicroRNAs (miRNAs) are small noncoding RNAs and have been showed to be associated with hepatic CSCs [52]. Therapy-resistant cancer stem cell exists that has the ability to reconstitute the tumor in its entirety [53]. Like normal stem cells, cancer stem cells should be rare, quiescent and capable of self renewing \& maintaining tumor growth and heterogeneity. Although the concept of cancer stem cells originates from that of normal stem cells [8]. Cell-based regenerative therapy is expected to be useful in non hematological diseases with cells of adult origin, such as mesenchymal stem cells (MSCs) and induced pluripotent stem cells (iPSCs) [54]. The appealing molecular and cellular properties of Mesenchymal Stem Cells (MSC), a type of adult stem cells have prompted the concept that these cells represent a reliable candidate for cell therapy [55]. Hematopoietic stem cells were widely used in transplantation experiments, especially in the treatment of leukemia and other cancers [56].

Allogeneic stem cell transplantation (SCT) can be considered the most successful method of adoptive immunotherapy of cancer. It is successful in part because of the potent graft-versus-tumor (GVT) effects of the donor graft, which are independent of the conditioning regimen [57]. Stem cells in mammary glands are located in the epithelium; canine mammary tumor cell line CMT-U229 has the property of forming duct-like structures in collagen gels which would be suitable for detection of tentative cancer stem-like cells [58]. Mechanistic possibilities for the therapeutic effects of stem cells include fusion with resident organ cells, immunomodulation and elicited through trophic mediators that result in the inhibition of fibrosis and apoptosis, enhancement of

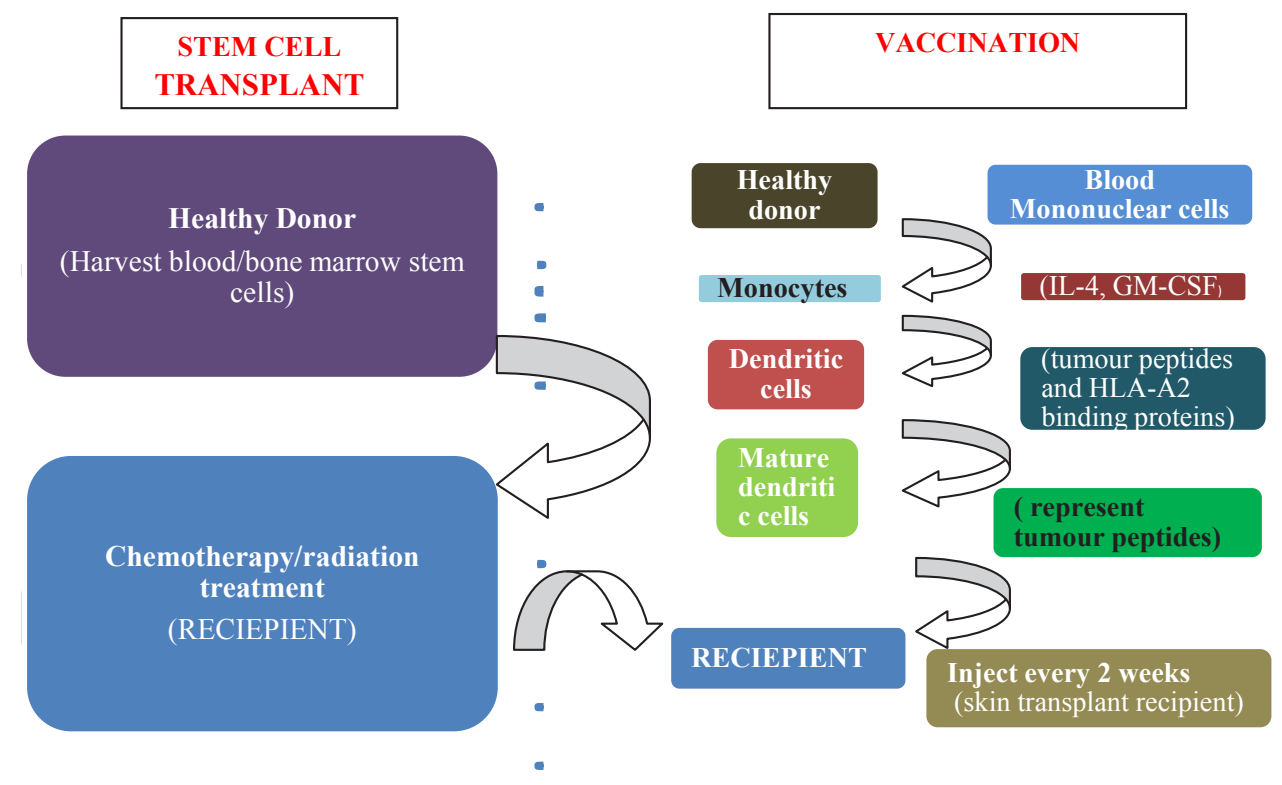

Figure 1: Stem cell and Vaccination process and there alternatives. 
angiogenesis, stimulation of mitosis and proliferation \& differentiation of organ-intrinsic precursor of stem cells [59]. The existence of cancer stem cells in gliomas and other brain tumours to therapy and relapse after standard treatments is attributed to the presence of stem-like cells. Glioma stem cells (GSCs) include properties such as multipotentiality, self-renewal, indefinite proliferation in vitro and tumorigenicity in vivo. stem cells always divided symmetrically found by means of a tumor sphere culture system and a single-cell subsphere generation assay that the majority C6 cells (>80\%) have stem cell properties [60]. Mesenchymal stem cells can be isolated from embryonic or adult tissues such as bone marrow and adipose tissue. Adipose-derived stem cells (ASCs) have a high proliferative capacity, and can enter the tumor vessels and differentiate into endothelial cells. This adipose derived stem cell induces protein SDF-1 on stage III tumours. This result in production of chemokines which results in tumour is present on the site. SDF1 may contribute to the production of other angiogenic factors such as IL-8 and MMP-3 by tumor cells [61]. Mammary gland cells predict to have the new stem cells including cells with stem cell-like properties. Studying the mouse mammary epithelial cells and indirectly, by studying development, morphogenesis, differentiation and carcinogenesis of xenotransplanted human breast epithelium in in vivo method. This similarity between mouse and human breast cells reveals the idea of prelocation of cancer cells and the effects of the surrounding cells after stem cells located [62][Figure 2].

\section{Discussion}

Tumor stem cells are poised to play an important role in the effort to achieve successful tumor ablation. Potential sources of this heterogeneity are genetic, epigenetic and environmental factors. The constitutional genomic features of individual patients (including gene copy number differences \& single nucleotide polymorphisms, among others) as well as somatic mutations /genetic instability features contribute to TSC heterogeneity. Along with the conventional treatments, new technologies, such as gene therapy \& nanotechnology could provide sophisticated multifunctional agents for simultaneous targeting, imaging, and therapy of TSCs [8]. Beside this some ethical issues are also concerned in production of the cancer stem cell. Introduction of chemokines on inducing stem cells to the tumour site leads the process of identification of cancer cells, but it takes a long process to identify and cure. Another question arise how academia, biotechnology and pharmaceutical companies use the information towards cancer eradication [63]. These are some ethical issues which rise during the treatment but according to the reports vaccination and stem methodology is the best way to cure the cancer.

The vaccine potentiality for curing the cancer depends upon the relativity of the antibodies in identifying the required epitopes. Some vaccines are microbial adjuvant, monoclonal antibodies and some are DNA vaccines. Vaccine depends upon some adjuvant for treatment; some are target specific and some bacterial toxins to prevent carcinogenicity of tumours. Even some are used after the different therapy process for the prevention of the side effects and to affect the cancerous cells which survive the therapy processes.

\section{Conclusion}

The review recites that stem cells and vaccination is the preventive method of curing the cancer. Many researchers and scientists have revealed the best way of curing cancer but there is lot to be discovered about the cancer. For the present scenario cancer stem cell will no doubt lead to new era of both basic \& clinical research, reclassification

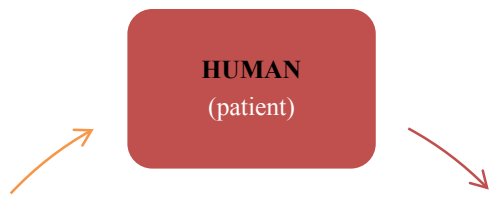

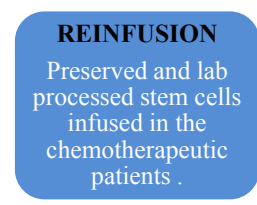
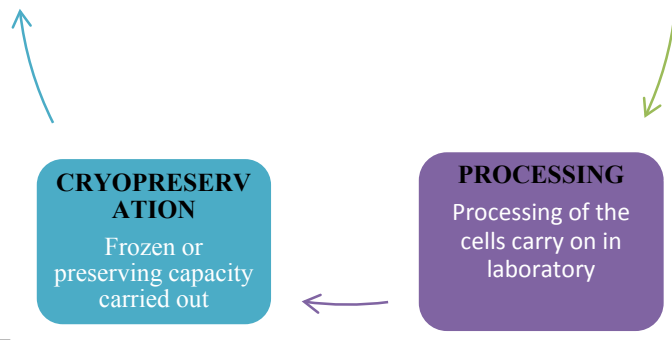
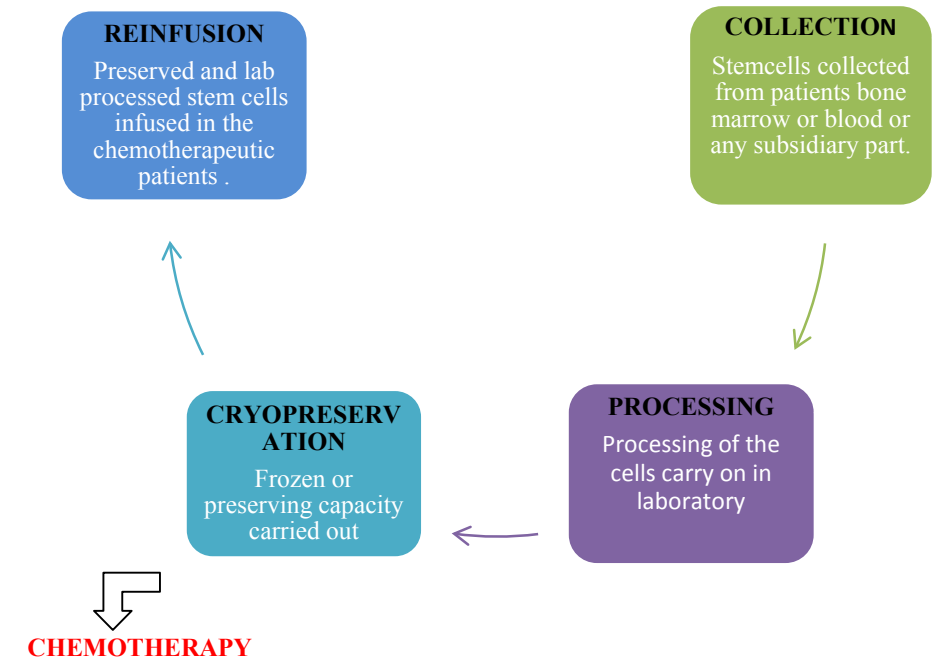

High dose chemotherapy/radiation

Survived patient

Figure 2: Stem cell therapy process. 
of human tumours \& development of novel therapeutic strategies specifically targeting cancer stem cells. Similary to increase the visibility of the vaccine. Steps should also be taken to educate providers in using required annual physicals process of utilization and the way it should be provided for the clinical trial. Surely stem cells and vaccines for cancer will be a boon in near future for the treatment of different cancer related diseases, it will help to eradicate it soon.

\section{References}

1. http://en.wikipedia.org/wiki/Cancer

2. Sudhakar A (2009) History of Cancer, Ancient and Modern Treatment Methods. J Cancer Sci Ther 1: 01-04.

3. http://www.ncbi.nlm.nih.gov/pubmedhealth/PMH0002287/

4. Vladimirova NM, Pisareva MA, Deineko NL, Bulycheva TI, et al. (2011) Tumor Specific Oligomeric Forms of Nucleophosmin. J Cancer Sci Ther 3: 205-212.

5. Naga Deepthi $\mathrm{CH}, \mathrm{VVL}$ Pavan Kumar A, Rameshbabu, Indirapriyadarshin $U$ (2011) Role of Tumor Suppressor Protein p53 in Apoptosis and Cancer Therapy. J Cancer Sci Ther 1: 01-06.

6. Debta P, Debta FM, Chaudhary M, Wadhwan V (2011) Evaluation of Prognostic Significance of Immunological Cells (Tissue Eosinophil and Mast Cell) Infiltration in Oral Squamous Cell Carcinoma. J Cancer Sci Ther 3: 201-204.

7. http://en.wikipedia.org/wiki/Multiple_myeloma

8. Shrihari TG (2011) Cancer Stem Cells - Therapeutic Boon!. J Cancer Sci Ther 3: $197-200$.

9. Porrata LF, Inwards DJ, Ansell SM, Micallef IN, Johnston PB, et al. (2011) Day 15 Peripheral Blood Lymphocyte/monocyte Ratio Post-autologous Peripheral Hematopoietic Stem Cell Transplantation and Survival in Diffuse Large B-cell Lymphoma. J Stem Cell Res Ther 1: 103.

10. http://en.wikipedia.org/wiki/Target_cell

11. http://en.wikipedia.org/wiki/Vaccine

12. http://en.wikipedia.org/wiki/Cancer_vaccine

13. Hu J, Schell TD, Peng X, Cladel NM, Balogh KK, et al. (2010) Using HLA-A2.1 Transgenic Rabbit Model to Screen and Characterize New HLA-A2.1 Restricted Epitope DNA Vaccines. J Vaccines Vaccin 1: 101.

14. Lee G, Cheung AP, Ge B, Zhu M, Li PP, et al. (2010) Monoclonal Anti-idiotype Antibodies against Carbohydrate-associate Epitope for Anti-Cancer Vaccine Development. J Vaccines Vaccin 1: 106

15. LaRocque JD, Berry-Cabán CS (2011) Human Papilloma Virus Vaccination Coverage among Soldiers in a Military Treatment Facility, 2007-2010. J Vaccines Vaccin 2: 116

16. http://en.wikipedia.org/wiki/Metastasis

17. Bonnet-Duquennoy M, Papon J, Mishellany F, Denoyer D, Labarre P, et al (2009) Promising Pre-clinical Validation of Targeted Radionuclide Therapy Using a [131I] Labelled lodoquinoxaline Derivative for an Effective Melanoma Treatment. J Cancer Sci Ther 1: 001-007.

18. Stajner I (2009) Cloudiness and Breast Cancer. J Cancer Sci Ther 1: 034-040.

19. Mi Z, Guo H, Markovic J, Kuo PC (2009) Characterization of Osteopontin Binding Kinetics In MDA-MB231 Breast and SK-Hep-1 Liver Cancer Cells. J Cancer Sci Ther 1: 047-051.

20. Dyavanagoudar SN (2009) Oral Submucous Fibrosis: Review on Etiopathogenesis. J Cancer Sci Ther 1: 072-077.

21. Miyazaki Y, Kikuchi K, González-Alva P, Inoue H, Noguchi Y, et al. (2010) Association of Butyric Acid Produced by Periodontopathic Bacteria with Progression of Oral Cancer. J Cancer Sci Ther 2: 026-032.

22. Shaghayegh K, Mahdi A, Ali K (2010) Larynx Preserving Treatments in the Early and Advanced Laryngeal Cancers; A Retrospective Analysis. J Cancer Sci Ther 1: 008-010.

23. Sarrazin S, Maurage CA, Delmas D, Lassalle P, Delehedde M (2010) Endocan as a Biomarker of endothelial Dysfunction in Cancer. J Cancer Sci Ther 2 : 047-052.

24. Singh RK, Sudhakar A, Lokeshwar BL (2010) Role of Chemokines and Chemokine Receptors in Prostate Cancer Development and Progression. J Cancer Sci Ther 2: 089-094.

25. Ammer AG, Kelley LC, Hayes KE, Evans JV, Lopez-Skinner LA, et al. (2009) Saracatinib Impairs Head and Neck Squamous Cell Carcinoma Invasion by Disrupting Invadopodia Function. J Cancer Sci Ther 1: 052-061.

26. Kingsley K, Zuckerman J, Davis M, Matteucci M, Knavel A, et al. (2009) Induction of Differential Growth in vitro by High-risk Human Papillomavirus in Human Breast Cancer Cell Lines is Associated with Caspase Dysregulation. J Cancer Sci Ther 1: 062-071.

27. Gayed IW, Wahba H, Wan D, Joseph U, Murthy R (2010) Effect of Y-90 SIRSpheres Therapy for Multiple Liver Metastases in a Variety of Tumors. J Cancer Sci Ther 2: 043-046.

28. Boosani CS, Varma AK, Sudhakar A (2009) Validation of Different Systems for Tumstatin Expression and its in-vitro and iv-vivo Activities. J Cancer Sci Ther 1: 008-018.

29. Allen JD, Chen M, Xie Y (2009) Model-Based Background Correction (MBCB) R methods and GUI for Illumina Bead-array Data. J Cancer Sci Ther 1: 025027.

30. Ishikawa T, Ishibashi J, Yamashita K, Dalkhsuren SO, Sumida K, et al. (2009) Non-Thermal Effects of Far-Infrared Ray (FIR) on Human Hepatocellular Carcinoma Cells HepG2 and their Tumors. J Cancer Sci Ther 1: 078-082.

31. Nayak BK, Krishnegowda NK, Galindo CA, Meltz ML, Swanson GP (2010) Synergistic Effect Between Curcumin (diferuloylmethane) and Radiation on Clonogenic Cell Death Independent of p53 in Prostate Cancer Cells. J Cancer Sci Ther 2: 171-181.

32. Karmakar S, Roy Choudhury S, Banik NL, Ray SK (2010) Activation of Multiple Molecular Mechanisms for Increasing Apoptosis in Human Glioblastoma T98G Xenograft. J Cancer Sci Ther 2: 107-113.

33. Müller CR, Namløs HM, Bjerner J, Østensen IHG, Sæter G, et al. (2010) Characterization of Treatment Response to Recombinant Interferon- $2 b$ in Osteosarcoma Xenografts. J Cancer Sci Ther 1: 016-025.

34. Shimul S, Rajiv G (2010) MTA1 Aids the AKT Pathway by Inhibiting Expression of a Key Regulator, PTEN. J Cancer Sci Ther 2: 114-119.

35. Avramidis D, Cruz M, Sidén Å, Tasat DR, Yakisich JS (2009) Regrowth Concentration Zero (RCO) as Complementary Endpoint Parameter to Evaluate Compound Candidates During Preclinical Drug Development for Cancer Treatment. J Cancer Sci Ther 1: 019-024.

36. Khan DR (2010) The Use of Nanocarriers for Drug Delivery in Cancer Therapy. J Cancer Sci Ther 2: 058-062.

37. Bruzzo J, Chiarella P, Ruggiero RA (2011) On the immunostimulatory hypothesis of cancer. Medicina (B Aires) 71: 509-513.

38. Galili U (2011) Conversion of Tumors into Autologous Vaccines by Intratumora Injection of $\alpha-G a l$ Glycolipids that Induce Anti-Gal/a-Gal Epitope Interaction. Clin Dev Immunol.

39. Balashova EE, Lokhov PG (2010) Proteolytically-cleaved Fragments of Cell Surface Proteins Stimulate a Cytotoxic Immune Response Against Tumoractivated Endothelial Cells In vitro. J Cancer Sci Ther 2: 126-131.

40. Tsimafeyeu I, Demidov L, Kharkevich G, Petenko N, Volkova M, et al. (2010) Granulocyte-Macrophage Colony-Stimulating Factor, Interferon Alpha and Interleukin-2 as Adjuvant Treatment for High-Risk Renal Cell Carcinoma. J Cancer Sci Ther 2: 157-159.

41. Vashistha S, Ajitkumar P (2011) Exposure to Interferon g Decreases Levels and Activity of Key Cell Cycle Proteins Resulting in Severe Growth Arrest of the Human Non-Transformed Cell Line, WISH. J Cancer Sci Ther 3: 013-019.

42. Wysong A, Asher SA, Yin X, Gore MR, Weinstein L, et al. (2011) Selective Inhibition of NF-kappa-B with NBD Peptide Reduces Tumor-Induced Wasting in a Murine Model of Cancer Cachexia In vivo. J Cancer Sci Ther 3: 022-029.

43. Nair LS, Mahesh S, Smitha LS, Sujathan K, Remani P (2011) Expressionof Canavalia gladiata lectin in leukemic cells. J Cancer Sci Ther 3: 088-091. 
Citation: Parthasarathi D, Palki D (2011) Vaccine Potentiality and Stem Cells Enhancing Cure to Cancer. J Cancer Sci Ther S17. doi:10.4172/19485956.S17-008

Page 8 of 8

44. Oliveira JP, Magliarelli HF, Pereira FV, Gianotti A, Soares-Costa A, et al. (2011) Sugarcane Cystatin CaneCPI-4 inhibits Melanoma Growth by Angiogenesis Disruption. J Cancer Sci Ther 3: 161-167.

45. Lechleider R, Pastan I (2011) Advances in the Development of Anti-CD22 Immunotoxins Containing Pseudomonas Exotoxin for Treatment of Hematologic Malignancies. J Cancer Sci Ther 3: 050-052.

46. Lara AR, Ramírez OT (2012) Plasmid DNA production for therapeutic applications. Methods Mol Biol 824: 271-303

47. Eccles SA (2011) The epidermal growth factor receptor/Erb-B/HER family in normal and malignant breast biology. Int J Dev Biol 55: 685-696.

48. Ahmed HG, Adam TM, Basama NK, Agabeldor AA (2011) Utility of CD3 and CD30 in Immunophenotyping of Lymphomas Among Sudanese Patients. J Cancer Sci Ther 3: 116-119.

49. Hopewell EL, Bronk CC, Massengill M, Engelman RW, Beg AA (2011) IKKßinduced inflammation impacts the kinetics but not the magnitude of the immune response to a viral vector. Eur J Immunol.

50. Decoster L, Wauters I, Vansteenkiste JF (2011) Vaccination therapy for nonsmall-cell lung cancer: review of agents in phase III development. Ann Oncol.

51. Canelles M (2011) The Universality of Asymmetric Division. J Stem Cell Res Ther 1: 1-2.

52. Ji J, Wang XW (2012) Identification of Cancer Stem Cell-Related MicroRNAs in Hepatocellular Carcinoma. Methods Mol Biol 826: 163-175.

53. Ghaffari S (2011) Cancer, stem cells and cancer stem cells: old ideas, new developments. F1000 Med Rep 3: 23.

54. Irioda AC, Zocche L, Souza CMCO, Ferreira RJ, Aliprandini E, et al. (2011) Pap
Test as the First Step in Screening Genetic Stability in Cell-Based Therapy. J Stem Cell Res Ther 1:106.

55. Minguell JJ, Pereira A, Bartholomew P and Lasala GP (2011) The Intrathecal Infusion of Mesenchymal Stem Cells into Healthy Rabbits is Safe and Devoid of Neurological or Clinical Complications. J Stem Cell Res Ther 1:104.

56. Meregalli M, Farini A, Torrente $Y(2011)$ Mesenchymal Stem Cells as Muscle Reservoir. J Stem Cell Res Ther 1: 105

57. Porter DL (2011) Allogeneic Immunotherapy to Optimize the Graft-versusTumor Effect: Concepts and Controversies. Hematology Am Soc Hematol Educ Program 2011: 292-298.

58. Ferletta M, Grawé J, Hellmén E (2011) Canine mammary tumors contain cancer stem-like cells and form spheroids with an embryonic stem cell signature. Int J Dev Biol 55: 791-799.

59. Abdel Aziz MT, Wassef MA, Rashed LA, Mhfouz S, Omar N, et al. (2011) Mesenchymal Stem Cells Therapy in Acute Renal Failure: Possible Role of Hepatocyte Growth Factor. J Stem Cell Res Ther 1:109.

60. Cruz M, Siden A, Tasat DR, Yakisich JS (2010) Are all Glioma Cells Cancer Stem Cells? J Cancer Sci Ther 2: 100-106.

61. Razmkhah M, Jaberipour M, Ghaderi A (2011) Chemokines and Chemokine Receptors Expression in the Adipose Derived Stem Cells (ASCs), Breas Tissues and in Peripheral Blood of Patients with Breast Cancer. J Carcinogene Mutagene 2: 120.

62. Fridriksdottir AJ, Petersen OW, Rønnov-Jessen L (2011) Mammary gland stem cells: current status and future challenges. Int J Dev Biol 55: 719-729.

63. Rameshwar P (2011) Post-identification of Cancer Stem Cell: Ethical and Scientific Dilemmas in Therapeutic Development?. J Stem Cell Res Ther 1: 1-2. 